\title{
Investigation of robustness of hybrid artificial neural network with artificial bee colony and firefly algorithm in predicting COVID-19 new cases: case study of Iran
}

Mohammad Javad Shaibani ( $\triangle$ Mjsheibani1993@gmail.com )

Tehran University of Medical Sciences https://orcid.org/0000-0003-0551-4433

Sara Emamgholipour

Tehran University of Medical Sciences

Samira Sadate Moazeni

Zahedan University of Medical Sciences

\section{Research Article}

Keywords: COVID-19, artificial neural network, artificial bee colony, firefly algorithm, hybrid model

Posted Date: August 13th, 2021

DOI: https://doi.org/10.21203/rs.3.rs-790321/v1

License: (c) (i) This work is licensed under a Creative Commons Attribution 4.0 International License.

Read Full License 


\title{
Investigation of robustness of hybrid artificial neural network with artificial bee colony and firefly algorithm in predicting COVID-19 new cases: case study of Iran
}

\author{
Mohammad Javad shaibani ${ }^{*}$, Sara Emamgholipour ${ }^{1,}$ Samira Sadate Moazeni ${ }^{2}$ \\ ${ }^{I}$ Department of Health Management and Economics, School of Public Health, Tehran University of Medical \\ Sciences, Tehran, Iran \\ ${ }^{2}$ Medical-Surgical Nursing Department, School of Nursing and Midwifery, Zahedan University of Medical \\ Sciences, Zahedan, Iran \\ *Corresponding Author: mjshaibani@ razi.tums.ac.ir
}

Abstract:

As an ongoing public health menace, the novel coronavirus pandemic has challenged the world. With several mutations and a high transmission rate, the virus is able to infect individuals in an exponential manner. At the same time, Iran is confronted with multiple wave peaks and the health care system is facing a major challenge. In consequence, developing a robust forecasting methodology can assist health authorities for effective planning. In that regard, with the help of Artificial Neural Network-Artificial Bee Colony (ANN-ABC) and Artificial Neural NetworkFirefly Algorithm (ANN-FA) as two robust hybrid artificial intelligence-based models, the current study intends to select the optimal model with the maximum accuracy rate. To do so, first a sample of COVID-19 confirmed cases in Iran ranging from 19 February 2020 to 25 July 2021 is compiled. $75 \%(25 \%)$ of total observation is randomly allocated as training (testing) data. Afterwards, an ANN models is trained with Levenberg Marquardt algorithm. Accordingly, based on R-squared and root-mean-square error criteria, the optimal number of hidden neurons is computed as 17 . The proposed ANN model is employed to develop ANN-ABC and ANN-FA models for achieving the maximum accuracy rate. According to $\mathrm{ANN}-\mathrm{ABC}$, the R- squared values of the optimal model are 0.9884 and 0.9885 at train and test stages, correspondingly. In respect to ANN-FA, for the selected model, the R-squared ranged from 0.9954 to 0.9940 at the train and test phases, respectively. The results indicated that both hybrid ANN-ABC and ANN-FA are the robust predictor of COVID-19 new cases in Iran. Additionally, with a slight difference, the ANN-FA model outperformed ANN$\mathrm{ABC}$ algorithm.

Keywords: COVID-19, artificial neural network, artificial bee colony, firefly algorithm, hybrid model. 


\section{Introduction}

As a severe acute respiratory syndrome, the novel coronavirus or COVID-19 (as stated by the World Health Organization) since its outbreak in December 2019 in Hubei Province of China, has altered the world. The pandemic was accompanied with the rapid transition conjointly with a huge disease burden. Because of that, the number of new positive cases had surged exponentially. Until August 2021, the total worldwide deaths attributed to COVID-19 has been more than 4.28 million (Worldometers dataset, 2021). Taking into consideration the speedy spread as well as the lifethreating outcomes of COVID-19, plenty of states put limits such as lockdown, wearing mask face or travel restrictions on normal life of citizens to reduce and manage the virus transition via behavioral change. The imposed limits stemmed from the crisis have considerable negative implications suchlike global economic recession (Fernandes, 2020), social stigma caused by mixed and misinformation (Sotgiu and Dobler, 2020), mental health issues including depression and anxiety (Xiong et al, 2020, Stankovska et al, 2020), educational concerns concerning quality and inequality (Marinoni et al, 2020), food insecurity concerns (Workie et al, 2020) or negative impact on quality of relationships (Pietromonaco et al, 2020), which overcome positive implications regarding ecological and environmental impacts (Verma and Prakash, 2020).

In the meanwhile, Iran has experienced several wave peaks since the initial announcement of COVID-19 as an epidemic on February 2020. As of the time of writing the current investigation, based on Worldometers dataset, the total cumulative death caused by COVID-19 in Iran has been 92,628 (Worldometers dataset, 2021). Mistrusts and cultural concerns regarding public polices (Jafari and Amiri Gharaghani, 2020), the financial and economic restriction for supporting quarantined people and entities (Yoosefi et al, 2021), the influence of economic sanctions on health sector to fight against COVID-19 (Takian et al, 2020) and dissemination of fake information and 
fallacious recommendations regarding pandemic on social media (Shalbafan and Khademoreza, 2020, Salimi et al, 2020) can be categorized as the bulk challenges of Iranian policy makers to battle against pandemic. In that sense, providing powerful predicational means to forecast the nearterm COVID-19 new cases can assist the health authorities to predict, select and evaluate the most promising policy instruments to control and alleviate the crisis. The remainder of this paper is ordered as follows: first we review the ongoing literature of COVID-19 forecasting. Then the modelling procedures used in this study including artificial neural network (ANN), artificial bee colony (ABC) and firefly algorithm (FA) will be described. After that, we will develop ANN, the hybrid ABC and FA models. Finally, the results will be presented and discussed.

\section{Literature survey}

From many years-ago, humankind was interested in forecasting multitudinous phenomena. In the contemporary era, the future trend prediction with minimum error (or maximum archivable accuracy) can represent a robust tool for policy making. Formulated on logical expectations of experts, there are qualitative forecasting methods. Still and all, those qualitative expectations might not provide a precise tool; Hence, these methods should be accompanied with quantitative tools. As a robust quantitative tool, artificial intelligence (AI)-based methods including Artificial Neural Network (ANN), Artificial Bee Colony (ABC) and Firefly Algorithm (FA) has archived various application in mining sciences (Koopialipoor et al (2019), Noroozi Ghaleini et al, 2019), biochemical engineering (Yeh and Hsieh, 2012), water quality forecasting (Chen et al, 2018), the optimal use of energy in residential buildings (Zhou et al, 2020), wind speed forecasting (Jia et al, 2016) as well as other computational fields. Nevertheless, as far as we are aware, employing ABC and FA algorithms for predicting COVID-19 cases is still limited. Despite this, many researchers have conducted investigations concerning COVID-19 trend forecasting via employing various 
research methodologies. There are many articles which applied regression and machine learning analysis using timeseries data. Parbat and Chakraborty (2020) focused on prediction COVID-19 new cases in India. They developed a vector regression model in professional software via considering the number of daily cases as dependent variable and number of observations (days) as independent variable. For forecasting the daily new cases, their results demonstrate $87 \%$ accuracy. Gothai et al (2021) have reported worldwide COVID-19 prediction using machine learning algorithms. They used linear regression, support vector machine and Holt's winter linear model. With an accuracy of $87 \%$, the Holt method outperformed the other models. Another machineleering-based prognostication is presented by Zivkovic and colleagues (2021). The COVID-19 new cases are predicted via a hybrid machine learning and beetle antennae search algorithms. They validate the proposed technique by using China and USA data. With a R-squared of 0.9763 , the authors concluded that the proposed model can be a robust forecaster of COVID-19 cases. Zheng et al (2020) proposed another AI-based hybrid model. They developed a susceptible-infected model for approximating infection rates. Then by using natural language processing module and long short-term memory as well as the improved susceptible-infected method, a hybrid model is developed. Additionally, the authors set the accuracy of traditional epidemic models as the benchmark and then compared it with the accuracy of the hybrid model. The results showed that the hybrid model can predict the COID-19 outbreak with less error. Khalilpourazari and Doulabi (2021) have also developed a hybrid machine learning algorithms as a forecaster of COVID-19 in Quebec, Canada. They developed a hybrid reinforcement learning based model and asses its accuracy. The mean square error is computed as $6.29 \mathrm{E}-06$. By using gradient-based grey wolf optimizer, Khalilpourazari et al (2021) have published a study concerning COVID-19 prediction. First, they compared their algorithm with some benchmark algorithm and concluded that the 
proposed model is more accurate. Then, via applying US data they predict that the COVID-19 cases will reach to 19 million by November, 2021. In the case of Iran, Behnam and Jahanmahin (2021) have predicted COVID-19 new cases using machine-learning methods and timeseries data. Based on the results, gaussian functions is the desirable predictor and they concluded that the peak of pandemic can occur 150 days after outburst. Talkhi et al (2021) have also modeled COVID-19 new cases in Iran. The authors compared various timeseries methods namely ARIMA, HoltWinter, prophet, multilayer perceptron model and extreme learning machine. The results indicated that with a root mean square error of 12.3 , the Holt-winter model can be a good predictor of COVID-19 deaths cases in Iran. Kafieh et al (2021) provided a similar study concerning COVID19 trend forecasting in Iran as well as other countries. The results demonstrated that with a root mean square error of 458.12, the multivariate long short-term memory is the optimal choice. The other study which focused on the worldwide prediction is reported by Yu and colleagues (2021). The authors employed various AI-based models namely autoregressive integrated moving average (ARIMA), feedforward neural network or long short-term memory (LSTM). Accordingly, they conclude that the results obtained from the deep learning approaches cannot be stable (i.e., for the most countries LSTM can provide a more promising prophecy). Furthermore, a comparative analysis of artificial neural network (ANN) and Gompertz technique for predicting the number of COVID-19 deaths in Mexico is reported by Conde-Gutiérrez and Colorado (2021). As a result of their study, by employing 10 coefficients it is observed that while ANN can fit real data with more accuracy the Gompertz technique with 3 parameters can be converged faster. Kolozsvari et al (2021) used AI-based methods to predict the epidemic curve of COVID-19 as well. Using global data, they have forecasted persistent peaks on the epidemic curve. In a further investigation, Shaharudin et al (2021) applied recurrent forecasting singular spectrum analysis to predict near- 
term COVID-19 cases in Malaysia. With a root mean square error of 19.2, they select the optimal model. A study conducted by Namasudra et al (2021) proposed nonlinear autoregressive neural network time series for predicting COVID-19 cases. They trained the model with various algorithms. The results indicated that Levenberg Marquardt training algorithms is the most suitable technique for training the neural network. Using multiple linear regression analysis as well as data of COVID-19 cases and number of phone calls of National Health Service 111 (NHS 111), Rostami-Tabar and Rendon-Sanchez (2021) have forecasted the daily COVID-19 cases in the UK. Compared to benchmark models (e.g., ARIMA, exponential smoothing or seasonal naïve), the authors concluded that with $95 \%$ of forecasting intervals for every horizon, their model can generate a more accurate prediction of COVID-19 cases in the UK. By employing online medical data in China (Shenzhen city), Huang and colleagues (2021) tried to forecast COVID-19 cases. They have used data regarding consultation, appointment and online outbreak search, and then conducted a multivariate vector autoregression analysis. The results revealed that online medical appointment can be a determinant factor in forecasting of COVID-19 cases up to two days. In another study, Guleryuz (2021) by deploying time series data of Turkey has presented COVID-19 trend prediction based on a univariate ARIMA, exponential smoothing and long short-term memory methods. Using Augmented Dickey Fuller, the author tested for stationary. The results showed the first difference of COVID-19 new cases will be stationary and ARIMA model outperforms the other methods. A similar result obtained from a study conducted by Alzahrani et al (2020) which used ARIMA to predict new cases in Saudi Arabia. They concluded that ARIMA outperforms autoregressive, moving average and integrated ARMA. A comparison of ARIMA with prophet forecasting model was made by Satrio et al (2021) in Indonesia. They concluded that prophet model is more precise than ARIMA. Nonetheless, by increasing time of prediction, the 
discrepancies between actual data and the prophet model prediction tend to increase. Roy et al (2021), Ceylan (2020), Awan and Aslam (2020), Malki et al (2021), Alabdulrazzaq et al (2021), Arora et al (2021) and ArunKumar et al (2021) have also predicted COVID-19 trends in different countries implementing ARIMA-based models.

As it was reviewed, there are various forecasting methods. Many researchers have utilized the ARIMA models as the main prediction technique or as a comparator for benchmark testing. It should be emphasized that ARIMA and other linear regression methods are not suitable in considering non-linear patterns among parameters. Thus, one can expect that the aforementioned models abate the accuracy rate as opposed to AI-based models since the AI techniques intend-to consider the non-linear relationships with a formidable and flexible discerning eligibility. Indispersion-through AI-based models, the hybrid ones usually enhance the accuracy rates; To rephrase it, the output of an AI-based model (i.e., ANN) is re-trained via a new training algorithm in order to increase the accuracy rate and optimize the wights attained by ANN. Thereby, one can anticipate that the hybrid models generate a robust forecasting of an output variable. In that regard, this study aims to forecast the COVID-19 new cases of Iran by virtue of Artificial Neural NetworkArtificial Bee Colony (henceforward ANN-ABC) and Artificial Neural Network-Firefly Algorithm (henceforth ANN-FA) as two robust models for acquiring the maximum archivable accuracy rate. Developed on our initial conjectures, we express the null hypothesizes as following: $H_{0}$ : There is no difference in forecasting robustness of ANN with ANN-ABC and ANN-FA.

$H_{0}$ : There is no difference in forecasting robustness of $A N N-A B C$ and ANN-FA models.

The major contributions of this study can be seen as providing a comparative and comprehensive analysis of the accuracy rates of two robust hybrid models in forecasting COVID-19 outbreak. 
Additionally, it is desired to supply the most promising forecasting route for Iranian health authorities to prognosticate the forthcoming trend of the COVID-19 pandemic. Hence, we expect that our proposed models can be served as an auxiliary policy instrument to periodically update the pandemic trend for a higher quality policy making in Iran.

\section{Research methodology}

\section{Artificial neural network}

Initially, the ANN models were developed via inspiring from biological neural network. In fact, ANN mimics the data processing procedure in the human brain and can learn from the surroundings. Because of their adaptivity, these models can solve nonlinear and complex problems with a greater velocity. Each ANN encompasses an input layer, hidden (middle) layer and an output layer. Neurons as the processing units, configure the bulk architectonic of an ANN. Hence, ANN consists of various sequential and parallel layers with interrelated nodes and neurons. The information is passed through synapses. Likewise, the items are connected to each other by using specific patterns. Conventionally, each node receives multiple inputs and combine them for representing its output. During generating the desire output, the network must be trained via a suitable algorithm (Maind and Wankar, 2014). Traditionally, back-propagation training process is widely used for arranging a feedforward network (Sazli, 2006). Since the output of each node can be the input of the subsequent node, in the training stage, first the generated output emits through the network. The ANN output is compared with the wished output. At this phase an error is disseminated. Then, by employing the network feedback (i.e., bias), the wights and bias can be adjusted. This process helps network to learn the existing pattern among input and output data (OMeara et al, 2018). In-the-interim, Multilayer Perceptron ANN with Levenberg-Marquardt training algorithm has gained substantial applications. Generally speaking, each Perceptron is a binary processing unit which classifies the achieved inputs. Then, the processed signals (inputs) 
will be directed for following Perceptron. For the purpose of establishing output, the weighted sum of incoming signals (inputs) must be processed. To do so, for the hidden and output layers, a stimulating function should be used. In that regard, the sigmoid transfer functions are mostly employed (Du and Stephanus, 2018). The produced output of every neuron is a function of weighted sum of the received inputs from the previous layers and an error value. Mathematically:

$$
\text { Output }_{\mathrm{j}}=\sum_{\mathrm{i}=1}^{\mathrm{i}=\mathrm{n}}\left(\mathrm{W}_{\mathrm{ij}} \mathrm{X}_{\mathrm{i}}+\mathrm{B}_{\mathrm{i}}\right)
$$

In E.q 1, i points to neuron $\mathrm{i}$ of the previous layer, $\mathrm{j}$ reffers to neuron $\mathrm{j}$ of the processing layer, $\mathrm{W}$ denotes wight and $\mathrm{B}$ stands for bias. As such, the activation faunction of neuron $\mathrm{Z}$ is obtained via following Sigmoid function:

$$
Z_{j}=\frac{1}{1+\exp \left(- \text { Output }_{j}\right)}
$$

Figure.1 displays an ANN modeling flow chart with one hidden layer.
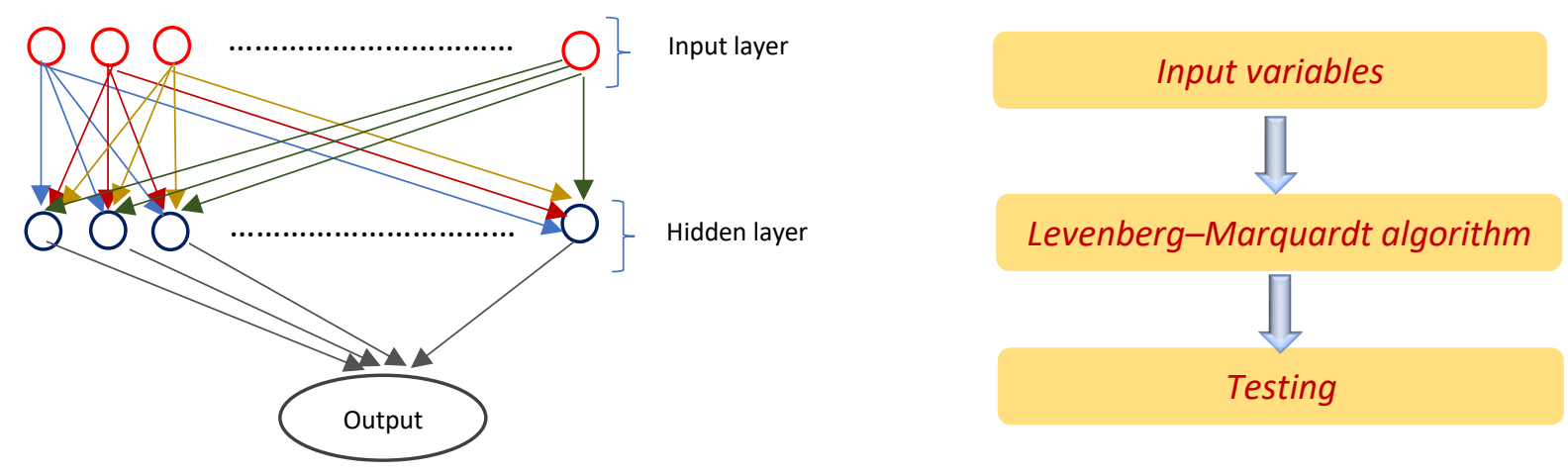

Figure 1. ANN modeling procedure

\section{Artificial bee colony}

Inspired from collective behavioral of colonies, many AI-based models have been developed. The $\mathrm{ABC}$ algorithm is devised by Karaboga (2005) grounding on collective knowledge of honeybees. Otherwise stated, by division of labor each member of a hive has a simple job. Nonetheless, when 
these members collaborate with each other, they generate a complex behavior (Karaboga, 2005). The ultimate goal of hive's members adapted to be as collecting the nectar of flowers. The rich nectar sources can be perceived in the form of optimal choices. Evidently, sharing information respecting nectar quantity of each source will be imperative for maximizing the nectar collection. Specifically, in $\mathrm{ABC}$ algorithm there are three types of bees which comprise scout, employed and onlooker bees (Zabihi and Nasiri, 2018). Each has a specific task:

Scout bees: Initially, a group of unemployed bees are chargeable for randomly searching new nectar sources. When the food source is examined and some nectar is collected, the bees move back into the hive. Via performing a waggle dance, they share data concerning nectar location and its quantity. This paves the way for other bees to conduct an investigation through the forage site.

Employed bees: The employed bees are responsible for exploring the nectar sources. They collect nectar and data about site position as well as profitability of forages.

Onlooker bees: There are some observer bees which processing the shared information regarding the forage sources. Due to the data, with a certain probability, they select a suitable forage for exploiting.

All in all, the ABC algorithm includes the following stages (Kumar et al, 2019; Jiang et al, 2019; Zabihi and Nasiri, 2018): firstly, the colony is dived into two subgroups; Fifty percent of total bees can be considered as employed bees. The remaining half incorporates onlookers. In this step, for each nectar site (i.e., a feasible solution for the problem), there will be one employed bee. Secondly, the profitability (fitness value) of solutions must be determined. In this step, the recruited bees must search for new food sources (i.e., a more optimal solution). The new forage can be acquired using the following Eq: 


$$
\mathrm{S}_{\mathrm{di}}=\mathrm{O}_{\mathrm{di}}+\varphi_{d i}\left(\mathrm{O}_{\mathrm{di}}-\mathrm{R}_{\mathrm{ri}}\right)
$$

Where $\mathrm{S}$ stands for new solutions (sources), $\mathrm{O}$ is the old solutions, $\varphi$ denotes a stochastic figure between -1 and $1, \mathrm{~d}$ is a random vector between one and number of answers, as well as $\mathrm{R}_{\mathrm{r}}$ includes a series of random solutions for the problem. If the profitability of new solution (S) is greater than old solution (O), the new solution will be generated. Then the old answer is substituted by the new solution. Alternatively, the new answer can be overlooked and fined, where the fitness is lower than the old answer. Thirdly, as mentioned earlier, on the ground of shared data regarding site fitness, the onlooker bees select the new nectar reservoir with a probability. This can be formulated as follow:

$$
\operatorname{Prob}_{d}=\frac{\text { fitness }_{d}}{\sum_{n=1}^{S \mathrm{~N}} \text { fitness }_{\mathrm{n}}}
$$

Hence, when a solution is selected, a new source will be generated (in the vicinity). If the new solution outperforms the previous answer, it will be substituted. As such, a new solution will be discarded and fined, if it dominated by the previous answer (Noroozi Ghaleini et al, 2019). In stage four, after employed bees infringe the model's restriction (e.g., number of probing which is determined previously by a threshold limit), the employed bees with nonenhanced solutions are converted into scout bees (Kumar et al, 2019). By the definition, again, the scouts perform random exploring. Thusly, this simple method separates the rich solution from the sub-optimal ones via creating negative and positive feedbacks for the sub-optimal and optimal solutions, respectively. Figure 2 presents a modelling flowchart of $\mathrm{ABC}$ algorithm. 


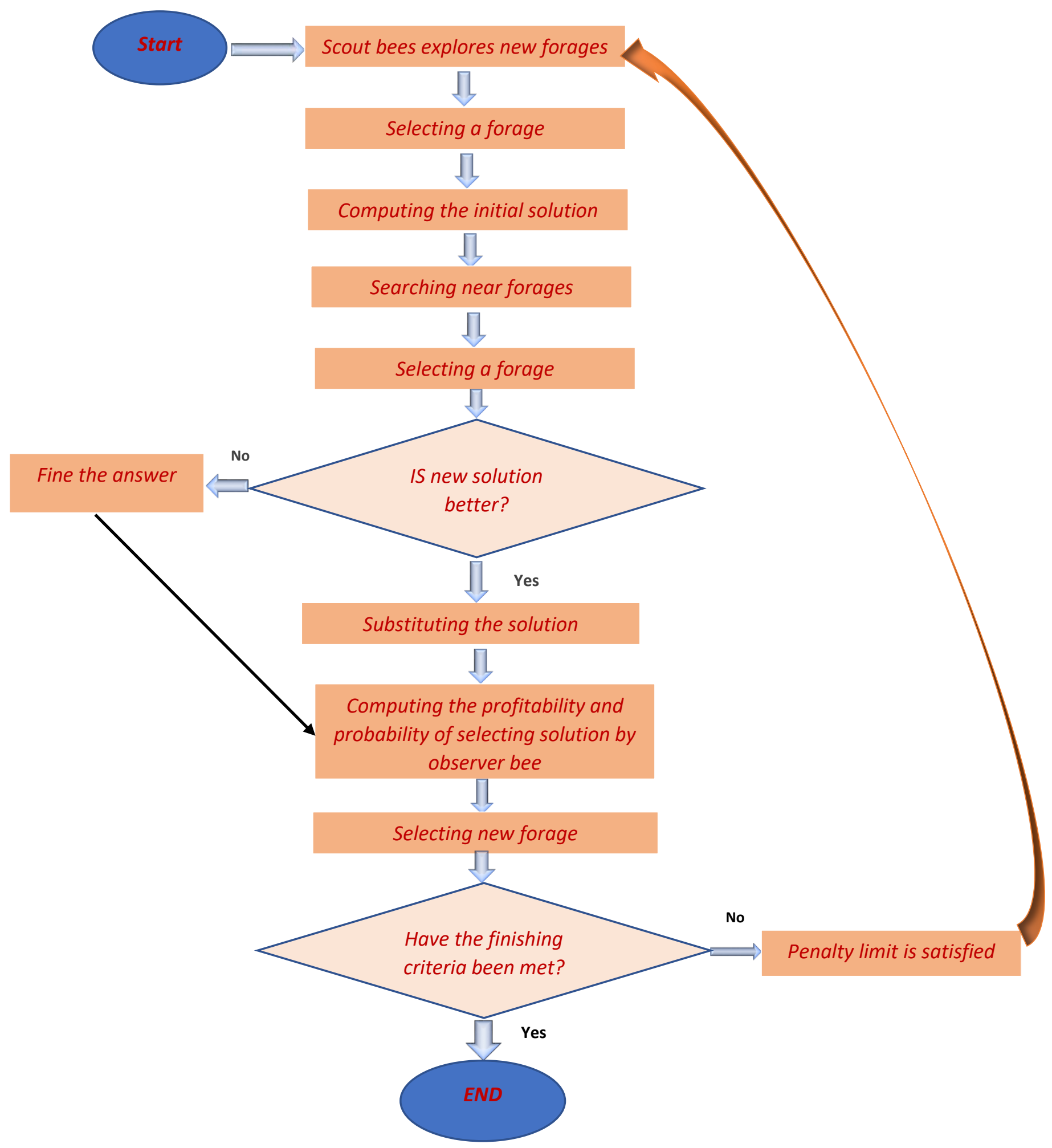

Figure 2. ABC modeling procedure 


\section{Firefly algorithm}

Initially, the firefly algorithm (FA) is introduced by Yang during 2007-2008. As a metaheuristicbased algorithm, FA tries to solve complex problems via inspiring from the social behavior of fireflies (Yang and He, 2013). These insects disseminate many lights with special patterns. This helps them to explore food, finding mate and associating with their own species (Koopialipoor et al, 2019). For the sake of modeling, the following assumptions should be considered (Yang and He, 2013):

- It is assumed that the sex of all fireflies is similar. Thus, there will be no ground for sextual attractiveness between insects.

- The amount of flashing lights is commensurate with their attractiveness. Additionally, it is hypothesized that there will be an inverse relationship between emitted lights (and consequently attractiveness) and fireflies' distance from each other. This means that if there exists a brighter firefly, the faint ones will be attracted to it. In the case of absenteeism of the shinier, fireflies maneuver in a randomly manner.

- The brilliancy of each firefly is influenced by an objective function.

In FA, based on physic principles, the light intensity of insects is incorporated with an objective function (Wahid et al, 2019). To formally express the model, let $\gamma, \beta, \beta_{0}, \mathrm{~d}$ and $\mathrm{x}$ be the absorption coefficient of light, attractiveness diversity, attractiveness where distance is zero, distance and fireflies' motion respectively. According to Yang and He (2013), since the attractiveness of a firefly is a function of light intensity perceived by other neighboring fireflies, $\beta$ can be defined as:

$$
\beta=\beta_{0} \mathrm{e}^{-\gamma \mathrm{d}^{2}}
$$


As such, the distance of firefly $\mathrm{i}$ from $\mathrm{j}$ is defined as the Cartesian distance (i.e., $\left\|\mathrm{X}_{\mathrm{i}}-\mathrm{X}_{\mathrm{j}}\right\|$ ). Thus, the motion of firefly $\mathrm{i}$ in-the-direction-of the brighter one $\mathrm{j}$ can be written as:

$$
\Delta \mathrm{X}_{\mathrm{i}}=\beta\left(\mathrm{X}_{\mathrm{j}}^{\mathrm{t}}-\mathrm{X}_{\mathrm{i}}^{\mathrm{t}}\right)+\alpha_{\mathrm{t}} \varepsilon_{\mathrm{i}}^{\mathrm{t}}
$$

In Eq.6, $\alpha$ and $\varepsilon$ are the randomization parameter and a random vector of numbers attained by a routine Gaussian function or other distribution like Levy flights (Yang and He, 2013). Via searching into literature, it is stated that a $\beta_{0}$ equals to one, an Alpha $\in[0,1]$ as well as $\gamma \in[0.1$, 10] are the most suitable values for the simulation (Koopialipoor et al, 2019). The modeling flowchart is depicted by Figure 3.

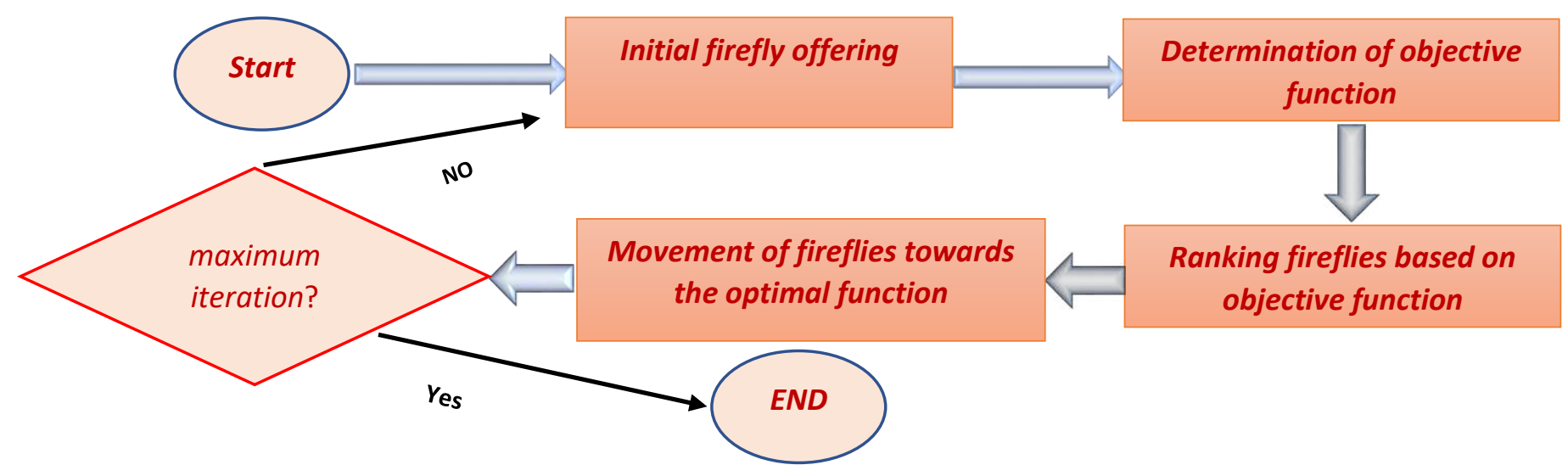

Figure 3. FA modeling procedure

\section{Evaluation criteria}

To evaluate the robustness of models, coefficient of determination $\left(\mathrm{R}^{2}\right)$ and root mean square error (RMSE) are employed. Statistically:

$$
\begin{gathered}
\mathrm{R}^{2}=\frac{\sum_{\mathrm{i}=}^{\mathrm{n}}\left(\mathrm{Y}_{\mathrm{i}}^{\sim}-\mathrm{Y}^{-}\right)^{2}}{\sum_{\mathrm{i}=}^{\mathrm{n}}\left(\mathrm{Y}_{\mathrm{i}}-\mathrm{Y}^{-}\right)^{2}} \\
\mathrm{RMSE}=\sqrt{\frac{\sum_{\mathrm{i}=}^{\mathrm{n}}\left(\mathrm{Y}_{\mathrm{i}}^{\sim}-\mathrm{Y}_{\mathrm{i}}\right)^{2}}{\mathrm{n}}}
\end{gathered}
$$


Where $\mathrm{Y}$ denotes actual value, $\mathrm{Y}^{\sim}$ stands for anticipated value, $\mathrm{Y}^{-}$is an average of actual values and lastly, $\mathrm{n}$ is the total number of observations. Obviously, a higher value of $\mathrm{R}^{2}$ will demonstrate more predictability power. Conversely, a lower value of RMSE will guarantee the robustness of the developed models.

\section{Developing hybrid models}

In the configuration of this paper, two hybrid models including ANN-ABC and ANN-FA are developed. Firstly, an ANN is developed in MATLAB software. After initial training, for the sake of hybridizing, the model is re-trained with the new algorithms. The hybrid modeling perdure is presented by Figure 4. The daily COVID-19 new cases are set as output data. Also, since the input data are unknown or may not be existed, the number of days (time) is considered as input instances (Tamang et al, 2020; Rustam et al, 2020). A sample of 523 daily observation at intervals 19 February 2020 and 25 July 2021 is collected from our world dataset. 392 of daily observations (75\% of total output) are randomly used for training network. The robustness of models will be tested and validated with 131 observation (25\% of total dataset).

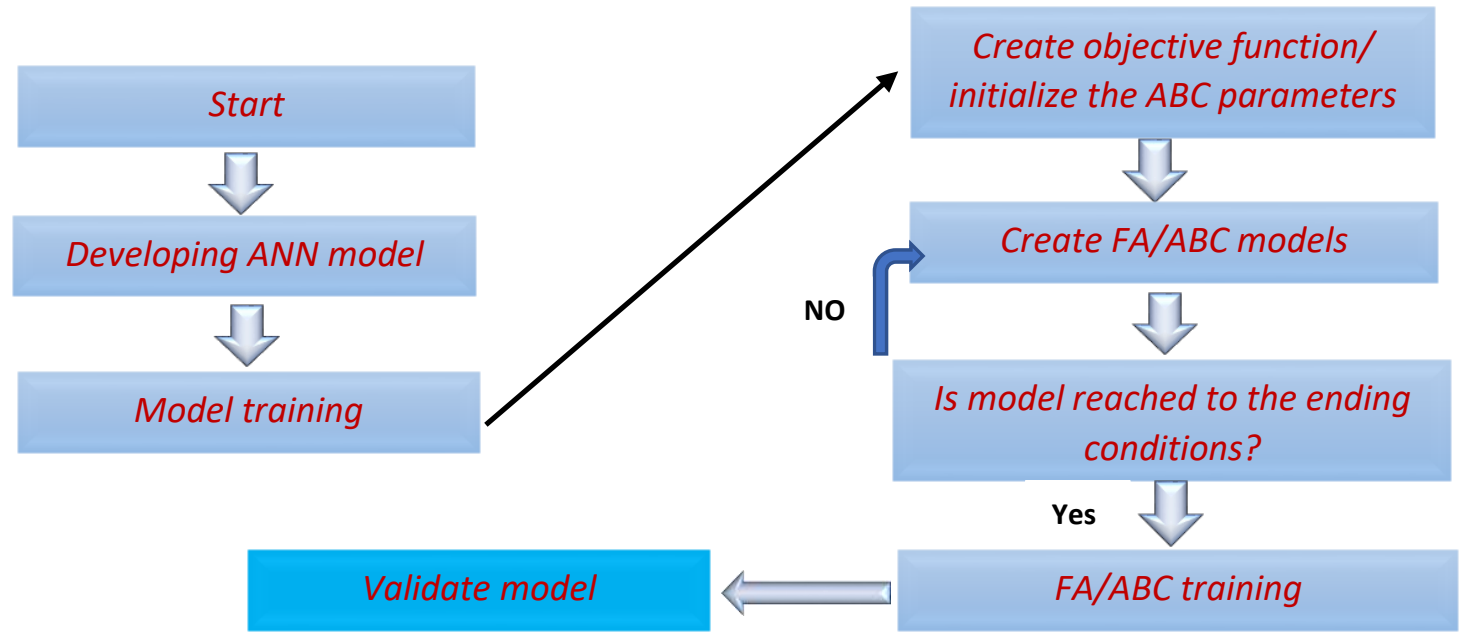

Figure 4. Hybrid modeling procedure 


\section{Results}

\section{Artificial neural network modeling}

In advance of hybrid modelling, first a Perceptron ANN with one hidden layer is developed. A single hidden layer will be adequate for tackling a sophisticate nonlinear problem (Karsoliya, 2012). Also, the Levenberg-Marquardt algorithm is selected for training the network. To specify the optimal number of hidden neurons, the routine procedure in the literature is followed (Koopialipoor et al, 2019, Noroozi Ghaleini et al, 2019, Zorlu et al, 2008). In that regard, 24 ANN models are developed. On account of electing the optimal alternative, each model is classified with respect to $\mathrm{R}^{2}$ and $\mathrm{RMSE}$. In respect to $\mathrm{R}^{2}$, a greater value is desired. Hence, in this ranking system, the model with maximum $\mathrm{R}^{2}$ has the highest score (i.e., 24 can be the maximum score). Inversely, a lower value of RMSE will be appropriate. Thus, the model with minimum value of RMSE gets the greatest score. Therefore, for each model the overall rank is attained by summing the two aforementioned figures for train and test stages, separately. Accordingly, in Table.1 the overall ranks attributable to the simulated models are calculated. As can be regarded, Model No. 16 with 17 neurons has acquired the maximum total rank. It can be claimed that $\mathrm{R}^{2}$ (RMSE) in this model reaches to its maximum (minimum) in the training stage. From this point on, $\mathrm{R}^{2}$ will decrease as the number of neurons increases. Subsequently, based on the overall rank, this model is picked as the optimal simulation. Figure. 5 compares the predicted COVID-19 new cases with actual cases in the training and testing stages (the values are normalized). As depicted, the proposed model has achieved a satisfactory accuracy rate throughout the testing phase. Nevertheless, given the dire consequences of COVID-19, improving the prediction accuracy rate will be unavoidable. In other respects, the ANN algorithms may not be able to find the optimal solution. Reaching to a local extremum cannot guarantee the optimization. The relative minimum trap shows that for example, RMSE is in its best possible value. However, in such cases, the utilization of a hybrid model assists 
in attaining the best possible value (i.e., the global extremum) (Noroozi Ghaleini et al, 2019).

Hence, in following the selected model is expanded into ANN-ABC and ANN-FA models. The results will be compared with ANN and the superior model will be preferred.

Table 1. Electing the optimal ANN model with respect to neurons

\begin{tabular}{|c|c|c|c|c|c|c|c|c|c|c|}
\hline \multirow[b]{2}{*}{ Items } & \multirow[b]{2}{*}{ Neurons } & \multicolumn{2}{|c|}{ Train } & \multicolumn{2}{|c|}{ Test } & \multicolumn{2}{|c|}{ Train } & \multicolumn{2}{|c|}{ Test } & \multirow[b]{2}{*}{$\begin{array}{c}\text { Overall } \\
\text { score }\end{array}$} \\
\hline & & $\mathrm{R}^{2}$ & RMSE & $\mathrm{R}^{2}$ & RMSE & $\begin{array}{c}\text { Rank: } \\
\mathrm{R}^{2}\end{array}$ & $\begin{array}{l}\text { Rank: } \\
\text { RMSE }\end{array}$ & $\begin{array}{c}\text { Rank: } \\
\mathrm{R}^{2}\end{array}$ & $\begin{array}{l}\text { Rank: } \\
\text { RMSE }\end{array}$ & \\
\hline 1 & 2 & 0.646 & 0.018 & 0.630 & 0.017 & 1 & 1 & 1 & 2 & 5 \\
\hline 2 & 3 & 0.664 & 0.016 & 0.634 & 0.022 & 2 & 2 & 2 & 1 & 7 \\
\hline 3 & 4 & 0.719 & 0.014 & 0.669 & 0.017 & 3 & 3 & 3 & 3 & 12 \\
\hline 4 & 5 & 0.731 & 0.014 & 0.717 & 0.014 & 4 & 4 & 4 & 4 & 16 \\
\hline 5 & 6 & 0.774 & 0.012 & 0.785 & 0.013 & 5 & 6 & 5 & 6 & 22 \\
\hline 6 & 7 & 0.787 & 0.010 & 0.799 & 0.014 & 6 & 5 & 6 & 5 & 22 \\
\hline 7 & 8 & 0.927 & 0.004 & 0.929 & 0.003 & 8 & 8 & 7 & 9 & 32 \\
\hline 8 & 9 & 0.924 & 0.004 & 0.929 & 0.004 & 7 & 7 & 7 & 7 & 28 \\
\hline 9 & 10 & 0.933 & 0.003 & 0.933 & 0.003 & 10 & 10 & 9 & 10 & 39 \\
\hline 10 & 11 & 0.931 & 0.003 & 0.931 & 0.003 & 9 & 9 & 8 & 13 & 39 \\
\hline 11 & 12 & 0.947 & 0.003 & 0.951 & 0.003 & 14 & 15 & 13 & 12 & 54 \\
\hline 12 & 13 & 0.945 & 0.003 & 0.949 & 0.003 & 13 & 14 & 12 & 8 & 47 \\
\hline 13 & 14 & 0.935 & 0.003 & 0.939 & 0.002 & 11 & 11 & 10 & 15 & 47 \\
\hline 14 & 15 & 0.945 & 0.003 & 0.945 & 0.002 & 13 & 13 & 11 & 17 & 54 \\
\hline 15 & 16 & 0.951 & 0.003 & 0.951 & 0.002 & 15 & 16 & 13 & 20 & 64 \\
\hline 16 & 17 & 0.962 & 0.002 & 0.972 & 0.002 & 20 & 24 & 19 & 23 & 86 \\
\hline 17 & 18 & 0.956 & 0.002 & 0.958 & 0.002 & 18 & 21 & 16 & 24 & 79 \\
\hline 18 & 19 & 0.958 & 0.002 & 0.964 & 0.002 & 19 & 23 & 18 & 18 & 78 \\
\hline 19 & 20 & 0.955 & 0.002 & 0.953 & 0.002 & 17 & 19 & 14 & 22 & 72 \\
\hline 20 & 21 & 0.955 & 0.002 & 0.956 & 0.002 & 17 & 20 & 15 & 16 & 68 \\
\hline 21 & 22 & 0.953 & 0.003 & 0.953 & 0.002 & 16 & 18 & 14 & 21 & 69 \\
\hline 22 & 23 & 0.956 & 0.002 & 0.962 & 0.003 & 18 & 22 & 17 & 11 & 68 \\
\hline 23 & 24 & 0.943 & 0.003 & 0.945 & 0.002 & 12 & 12 & 11 & 19 & 54 \\
\hline 24 & 25 & 0.951 & 0.003 & 0.951 & 0.003 & 15 & 17 & 13 & 14 & 59 \\
\hline
\end{tabular}



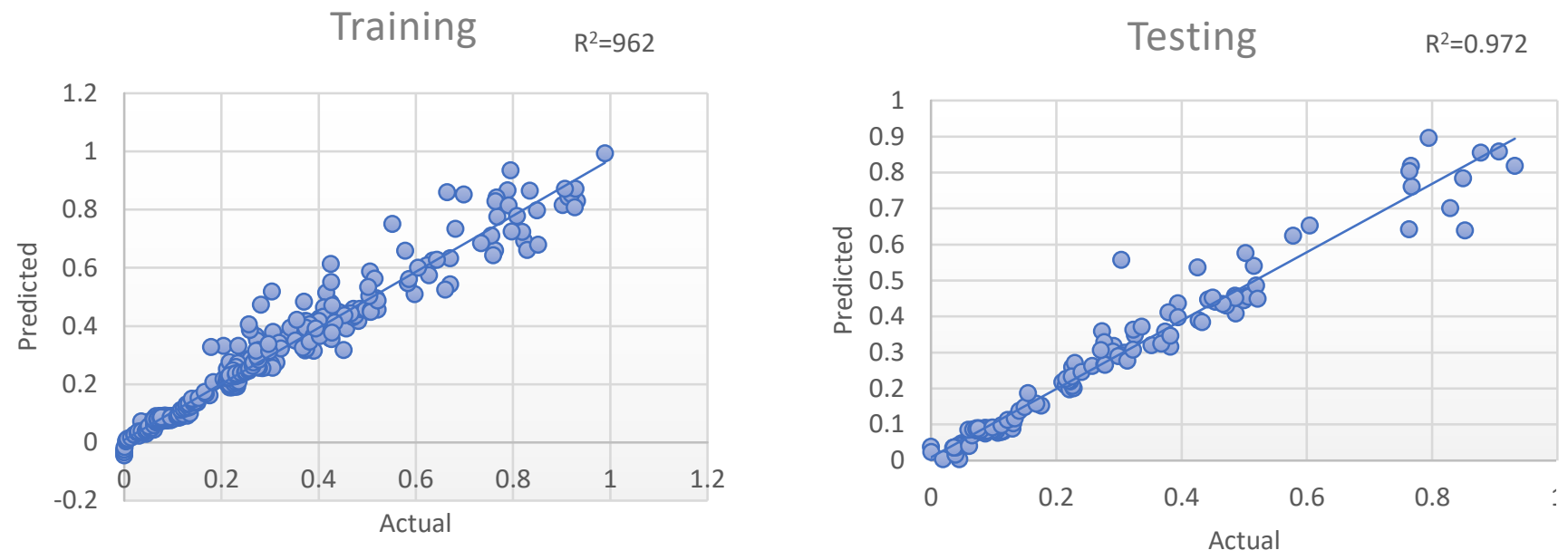

Figure 5. ANN optimal model performance in predicting COVID-19 new cases

\section{Developing hybrid artificial bee colony}

The ANN optimal prediction with 17 neurons is selected for developing ANN-ABC model. To do so, the optimum number of bees must be specified. It is a crucial stage, because as the number of bees increases, their effort to find the best solution also increases. As a logical expectation, the sub-optimal number of bees can decline the accuracy rate. On the other hand, a huge number of bees will increase computational time which may not be associated with a lower RMSE. Therefore, to find the desirable number of bees, the model is computed by regarding various number of bees and iterations. The results are indicated by Figure 6. The number of iterations is regarded 1000 and the bee's number is between 10 and 80 . With 300 repetitions, almost all series will be converged. As shown in Fig. 6, practically, for number of bees more than 40, the solution will not be improved. In this respect, the model with 40 bees is selected as the best ANN-ABC model. The results of predicting COVID-19 new cases with the proposed model, are depicted by Figure 7. Accordingly, in comparison with ANN, the ANN-ABC demonstrates a promising forecasting robustness in either train or test stages. 


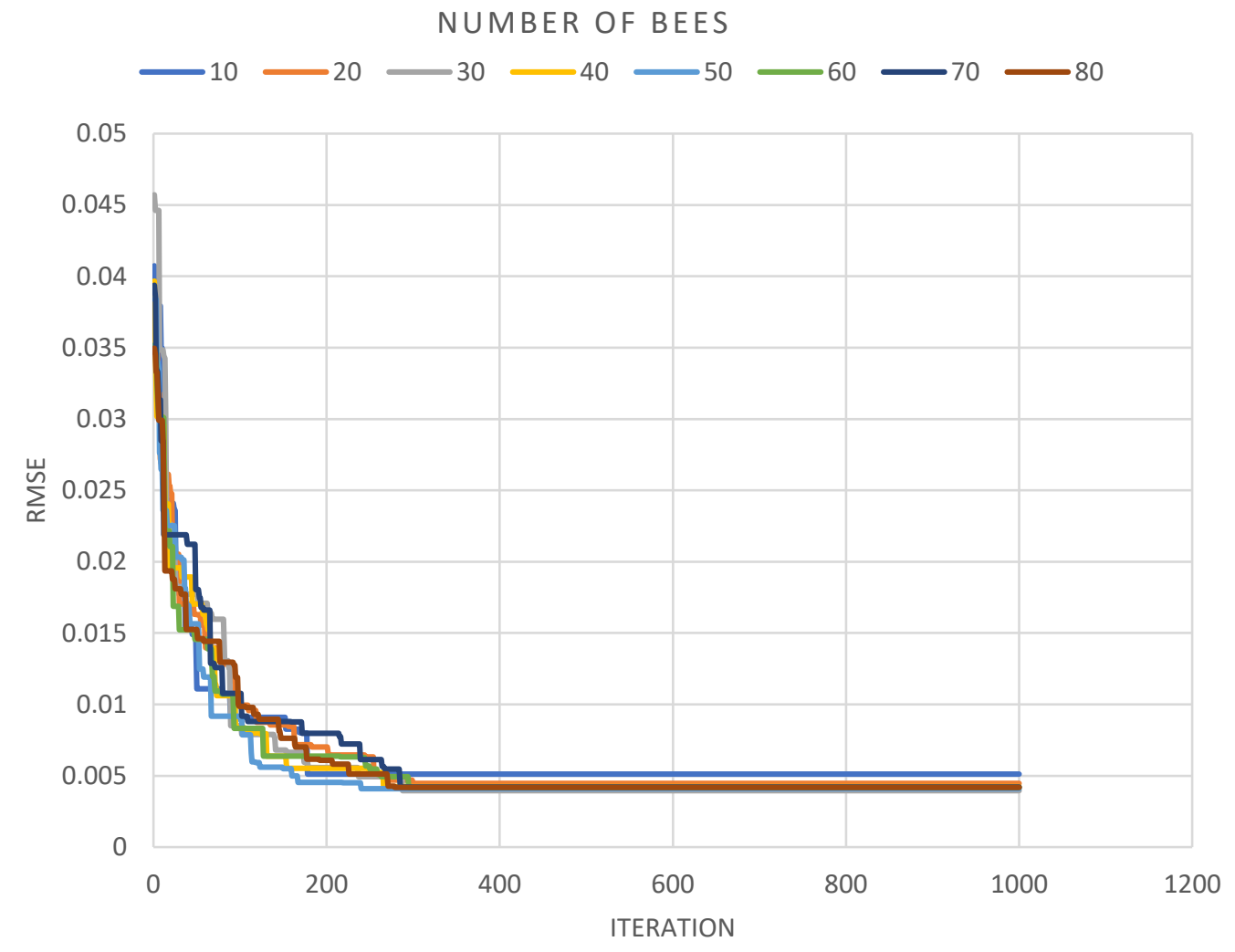

Figure 6. ANN-ABC models regarding number of bees and iteration
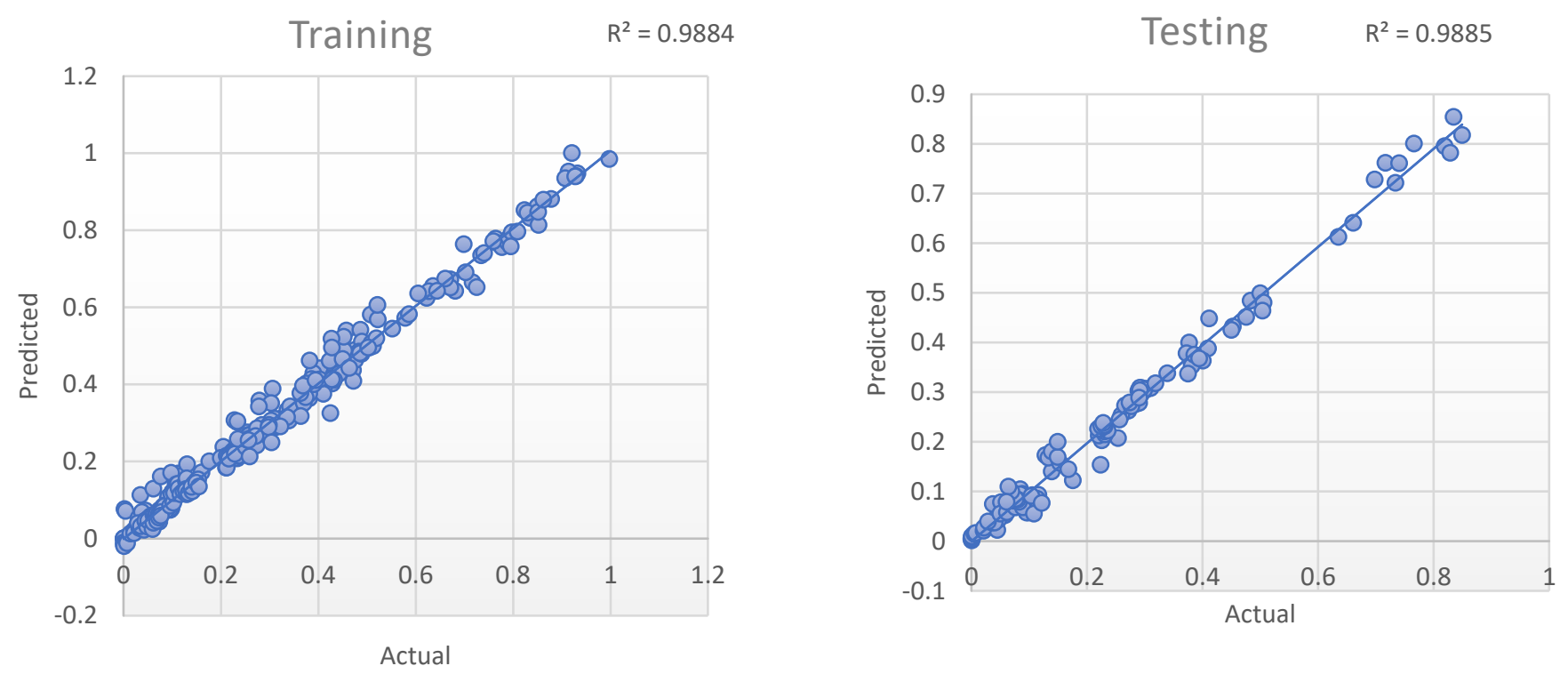

Figure 7. ANN-ABC optimal model performance in predicting COVID-19 new cases 


\section{Developing hybrid firefly algorithm}

As before, the proposed ANN model with 17 neurons is incorporated into FA. Generally, for optimizing the search area, the number of fireflies should be specified. Similar to ANN-ABC case, a large number of insects will increase the search area to find the optimal solution. However, this may increase the computational time. Thus, by considering 1000 repetition as well as the number of fireflies diverging from 10 to 80 , an investigation is conducted to establish the optimal model. As shown by Figure 8, approximately after 300 iterations the models are converged. In this case, the ANN-FA with 30 fireflies is the best (i.e., with more than 30 fireflies the computational time will be increased while the solution is almost the same). Considering the proposed ANN-FA model with 30 fireflies, Figure 9 displays the results. Correspondingly, with a R-squared more than $99 \%$ at both train and test stages the ANN-FA has obtained a promising robustness in approximating the COVID-19 confirmed new cases in Iran.

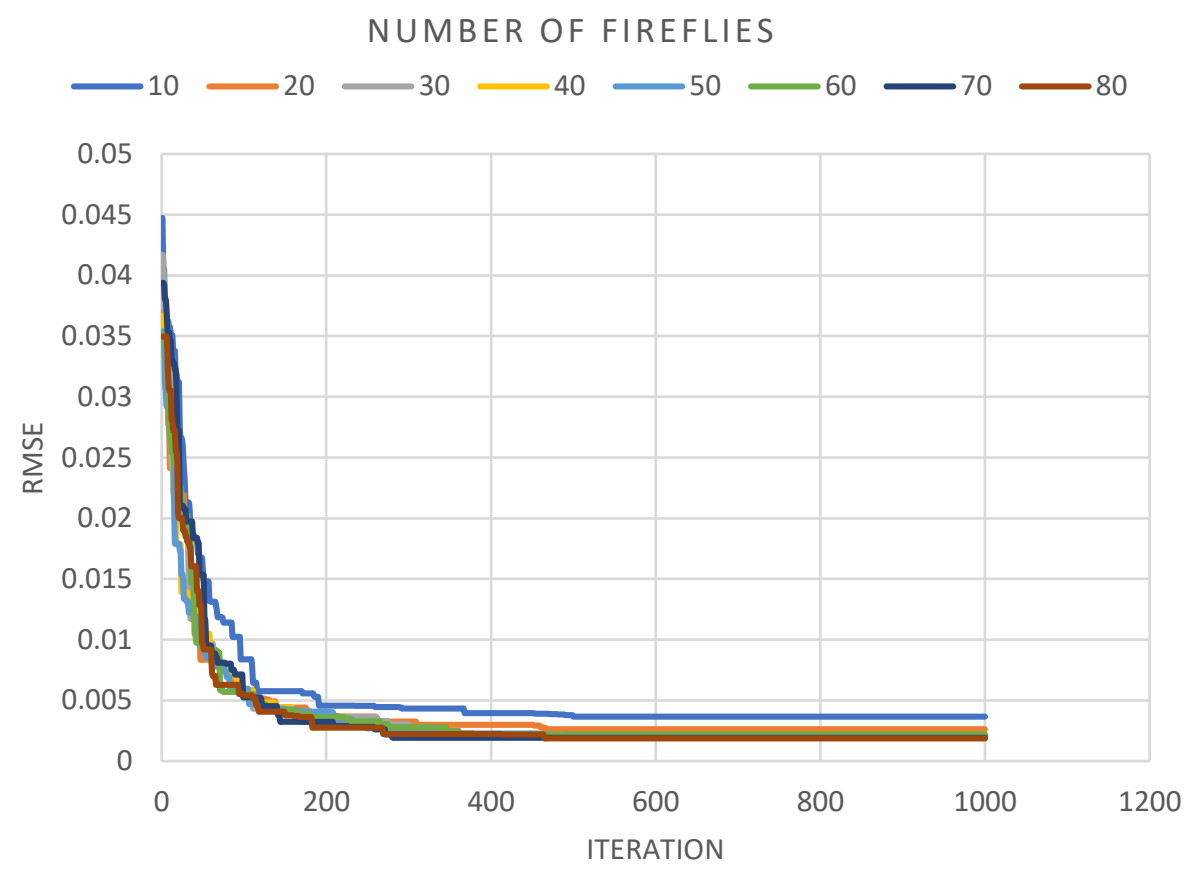

Figure 8. ANN-FA models regarding number of fireflies and iteration 

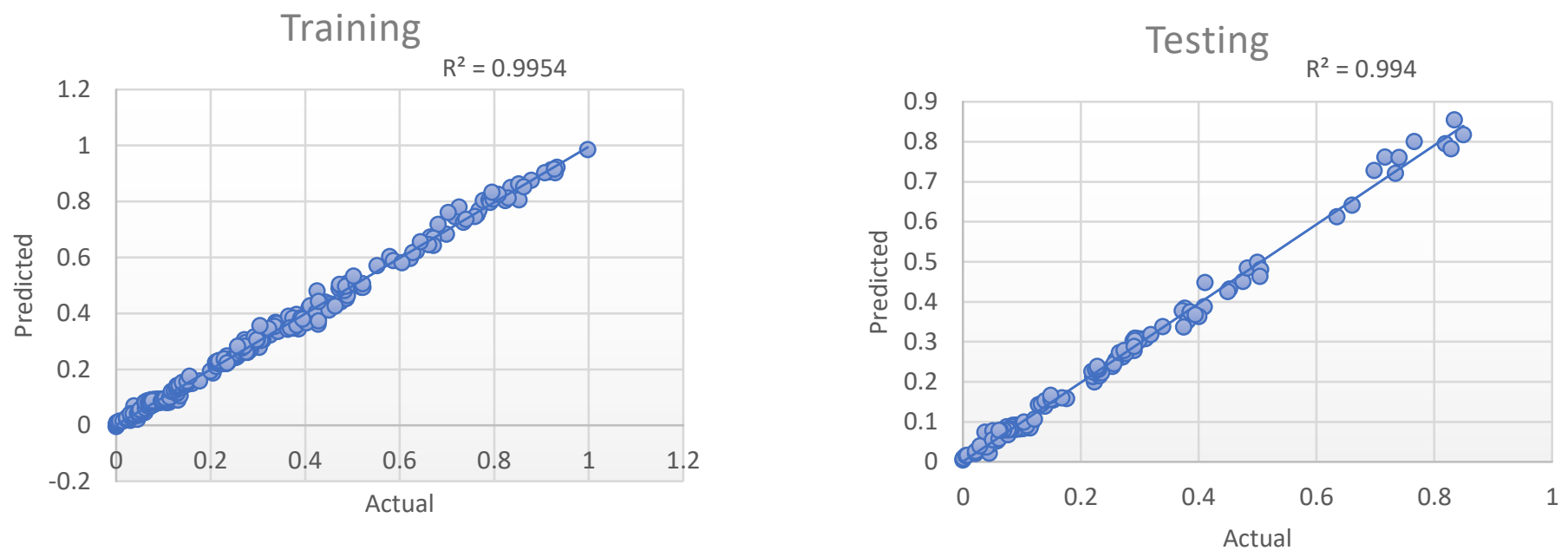

Figure 9. ANN-FA optimal model performance in predicting COVID-19 new cases

\section{Dissuasion and conclusion}

Presently, the COVID-19 pandemic with serious consequences and the rapid transition has involved Iran and other countries in crisis. Up till now, many researchers have provided various models including autoregressive integrated moving average, machine-learning methods, prophet models and other approaches for predicting the COVID-19 trends. Generally, each model has its pros and cons. Regarding the limitation of data, the objective of study and context, selecting the best model may not be possible per se. Currently, the hybrid ANN models have been utilized in various fields and showed a remarkable robustness. Hence, as part of this study, we proposed two hybrid ANN-based models for achieving the maximum forecasting robustness of COVID-19 new cases in Iran. To do so, first, a sample of 523 COVID-19 daily confirmed cases was divided randomly into train (75\%) and test (25\%) data. Then, we developed the ANN model. In this step, a model with 17 hidden neurons was selected for further development. It should be noted that the proposed ANN model showed a good predictability. Effectively, with a R-squared of 0.962 and 0.972 as well as a RMSE of.002057 and 0.001766 for the train and test steps, the proposed ANN model with Levenberg-Marquardt learning algorithm can be considered as a powerful tool for prediction of COVID-19 new cases in Iran. However, due to the relative extremum trap in ANN, 
the global extremum may not be reached. In that regard, the hybrid models namely ANN-ABC and ANN-FA can enhance the accuracy of ANN. Thus, we developed ANN-ABC and ANN-ABC to increase the accuracy rate and optimize the results obtained by ANN. For ANN-ABC modeling, first the optimum number of bees and iterations were identified as 40 and 300 respectively. The R-squared for both train and test stages were approximately 0.988 . Hence, compared to ANN model, the hybrid $\mathrm{ABC}-\mathrm{ANN}$ reinforced the forecasting robustness. Over and above that, we also developed the hybrid ANN-FA model. In this case, a model with 30 fireflies and almost 300 iterations, was selected. The R-squared of the train and test stages were 0.995 and 0.994 , respectively. To formally express these results, we reject the null hypothesizes. The two hybrid models outperformed the ANN; Thus, $H_{0}$ must be rejected. As the same way, the ANN-FA outperformed ANN-ABC; Therefore, $H_{0}{ }^{\prime}$ can be rejected. It ought to be remarked that such models should be used for short-term predicting. Otherwise stated, the actions or inactions of policymakers and the extent of public cooperation to control the disease, can adjust the outbreak trend in longer times. However, one of the strengths of our proposed models was their parsimony. They also can be used for scenario making suchlike vaccination or quarantine as long as their trends or impact on COVID-19 new cases could be initialized. In sum, according to the results, we conclude that both ANN-ABC and ANN-FA are fairly strong to forecast pandemic but ANN-FA will be the most promising technique for predicting COVID-19 new cases in Iran.

\section{Note on contribution}

All authors have participated into drafting. MJ.Shaibani and S.Emamgholipour performed software analysis. S.Sadate Moazeni had substantial contribution in developing ANN-FA.

\section{Acknowledgment}

The authors are grateful to Tehran University of Medical Sciences (TUMS) for providing access to the professional software and web data.

\section{Funding}


This study has not had any financial supports.

\section{Conflict of interests}

We have no conflict of interest to declare.

\section{Data availability statement}

Data used in the simulations can be found at https://ourworldindata.org/coronavirus-source-data.

\section{Code availability}

The source codes are inherently exclusive and might-be provided with constraints.

\section{Consent for publication}

We confirm that this research is original. Also, we have read this manuscript and agree with its dissemination.

\section{References}

Arora, R., Agrawal, A., Arora, R., Poonia, R. C., \& Madaan, V. (2021). Prediction and forecasting of COVID-19 outbreak using regression and ARIMA models. Journal of Interdisciplinary Mathematics, 24(1), 227-243. https://doi.org/10.1080/09720502.2020.1840075.

Awan, T. M., \& Aslam, F. (2020). Prediction of daily COVID-19 cases in European countries using automatic ARIMA model. Journal of Public Health Research, 9(3). https://dx.doi.org/10.4081\%2Fjphr.2020.1765.

Alabdulrazzaq, H., Alenezi, M. N., Rawajfih, Y., AlGhannam, B. A., Al-Hassan, A. A., \& AlAnzi, F. S. (2021). On the accuracy of ARIMA based prediction of COVID-19 spread. Results in Physics, 104509. https://doi.org/10.1016/j.rinp.2021.104509.

Alzahrani, S. I., Aljamaan, I. A., \& Al-Fakih, E. A. (2020). Forecasting the spread of the COVID19 pandemic in Saudi Arabia using ARIMA prediction model under current public health interventions. Journal of infection and public health, 13(7), 914-919. https://doi.org/10.1016/j.jiph.2020.06.001.

ArunKumar, K. E., Kalaga, D. V., Kumar, C. M. S., Chilkoor, G., Kawaji, M., \& Brenza, T. M. (2021). Forecasting the dynamics of cumulative COVID-19 cases (confirmed, recovered and deaths) for top-16 countries using statistical machine learning models: Auto-Regressive Integrated Moving Average (ARIMA) and Seasonal Auto-Regressive Integrated Moving Average (SARIMA). Applied soft computing, 103, 107161. https://doi.org/10.1016/j.asoc.2021.107161.

Behnam, A., \& Jahanmahin, R. (2021). A data analytics approach for COVID-19 spread and end prediction (with a case study in Iran). Modeling earth systems and environment, 1-11. https://doi.org/10.1007/s40808-021-01086-8.

Chen, S., Fang, G., Huang, X., \& Zhang, Y. (2018). Water quality prediction model of a water diversion project based on the improved artificial bee colony-backpropagation neural network. Water, 10(6), 806. https://doi.org/10.3390/w10060806. 
Conde-Gutiérrez, R. A., Colorado, D., \& Hernández-Bautista, S. L. (2021). Comparison of an artificial neural network and Gompertz model for predicting the dynamics of deaths from COVID19 in México. Nonlinear Dynamics, 1-15. https://doi.org/10.1007/s11071-021-06471-7.

Ceylan, Z. (2020). Estimation of COVID-19 prevalence in Italy, Spain, and France. Science of The Total Environment, 729, 138817. https://doi.org/10.1016/j.scitotenv.2020.138817.

Du, Y. C., \& Stephanus, A. (2018). Levenberg-Marquardt neural network algorithm for degree of arteriovenous fistula stenosis classification using a dual optical photoplethysmography sensor. Sensors, 18(7), 2322. http://dx.doi.org/10.3390/s18072322.

Fernandes, Nuno. "Economic effects of coronavirus outbreak (COVID-19) on the world economy." Available at SSRN 3557504 (2020).

Ghaleini, E. N., Koopialipoor, M., Momenzadeh, M., Sarafraz, M. E., Mohamad, E. T., \& Gordan, B. (2019). A combination of artificial bee colony and neural network for approximating the safety factor of retaining walls. Engineering with Computers, 35(2), 647-658. https://doi.org/10.1007/s00366-018-0625-3.

Gothai, E., Thamilselvan, R., Rajalaxmi, R. R., Sadana, R. M., Ragavi, A., \& Sakthivel, R. (2021). Prediction of covid-19 growth and trend using machine learning approach. Materials Today: Proceedings. https://dx.doi.org/10.1016\%2Fj.matpr.2021.04.051.

Guleryuz, D. (2021). Forecasting outbreak of COVID-19 in Turkey; Comparison of Box-Jenkins, Brown's exponential smoothing and long short-term memory models. Process Safety and Environmental Protection, 149, 927-935. https://dx.doi.org/10.1016\%2Fj.psep.2021.03.032.

Huang, W., Cao, B., Yang, G., Luo, N., \& Chao, N. (2021). Turn to the Internet First? Using Online Medical Behavioral Data to Forecast COVID-19 Epidemic Trend. Information Processing \& Management, 58(3), 102486. https://dx.doi.org/10.1016\%2Fj.ipm.2020.102486.

Jia, G., Li, D., Yao, L., \& Zhao, P. (2016, June). An improved artificial bee colony-BP neural network algorithm in the short-term wind speed prediction. In 2016 12th World Congress on Intelligent Control and Automation (WCICA) (pp. 2252-2255). IEEE. https://doi.org/10.1109/WCICA.2016.7578265.

Jafari, H., \& Gharaghani, M. A. (2020). Cultural challenges: The most important challenge of COVID-19 control policies in Iran. Prehospital and Disaster Medicine, 35(4), 470-471. https://doi.org/10.1017/S1049023X20000710.

Jiang, S., Lu, C., Zhang, S., Lu, X., Tsai, S. B., Wang, C. K., ... \& Lee, C. H. (2019). Prediction of ecological pressure on resource-based cities based on an RBF neural network optimized by an improved ABC algorithm. IEEE Access, 7, 47423-47436. https://doi.org/10.1109/ACCESS.2019.2908662.

Khalilpourazari, S., \& Doulabi, H. H. (2021). Designing a hybrid reinforcement learning based algorithm with application in prediction of the COVID-19 pandemic in Quebec. Annals of Operations Research, 1-45. https://doi.org/10.1007/s10479-020-03871-7. 
Khalilpourazari, S., Doulabi, H. H., Çiftçioğlu, A. Ö., \& Weber, G. W. (2021). Gradient-based grey wolf optimizer with Gaussian walk: Application in modelling and prediction of the COVID19 pandemic. Expert Systems with Applications, 177, 114920. https://doi.org/10.1016/j.eswa.2021.114920.

Kafieh, R., Arian, R., Saeedizadeh, N., Amini, Z., Serej, N. D., Minaee, S., ... \& Haghjooy Javanmard, S. (2021). Covid-19 in iran: Forecasting pandemic using deep learning. Computational and mathematical methods in medicine, 2021. https://doi.org/10.1155/2021/6927985.

Karaboga, D. (2005). An idea based on honey bee swarm for numerical optimization (Vol. 200, pp. 1-10). Technical report-tr06, Erciyes university, engineering faculty, computer engineering department.

Kolozsvari, L. R., Berczes, T., Hajdu, A., Gesztelyi, R., TIba, A., Varga, I., ... \& Zsuga, J. (2021). Predicting the epidemic curve of the coronavirus (SARS-CoV-2) disease (COVID-19) using artificial intelligence. MedRxiv, 2020-04. https://doi.org/10.1101/2020.04.17.20069666.

Kumar, S., Nayyar, A., \& Kumari, R. (2019). Arrhenius artificial bee colony algorithm. In International conference on innovative computing and communications (pp. 187-195). Springer, Singapore. https://doi.org/10.1007/978-981-13-2354-6_21.

Koopialipoor, M., Noorbakhsh, A., Noroozi Ghaleini, E., Jahed Armaghani, D., \& Yagiz, S. (2019). A new approach for estimation of rock brittleness based on non-destructive tests. Nondestructive Testing and Evaluation, 354-375. https://doi.org/10.1080/10589759.2019.1623214.

Karsoliya, S. (2012). Approximating number of hidden layer neurons in multiple hidden layer BPNN architecture. International Journal of Engineering Trends and Technology, 3(6), 714-717.

Marinoni, G., Van't Land, H., \& Jensen, T. (2020). The impact of Covid-19 on higher education around the world. IAU Global Survey Report.

Maind, S. B., \& Wankar, P. (2014). Research paper on basic of artificial neural network. International Journal on Recent and Innovation Trends in Computing and Communication, 2(1), 96-100.

Namasudra, S., Dhamodharavadhani, S., \& Rathipriya, R. (2021). Nonlinear neural network-based forecasting model for predicting COVID-19 cases. Neural Processing Letters, 1-21. https://doi.org/10.1007/s11063-021-10495-w.

ostami-Tabar, B., \& Rendon-Sanchez, J. F. (2021). Forecasting COVID-19 daily cases using phone call data. Applied soft computing, 106932. https://dx.doi.org/10.1016\%2Fj.asoc.2020.106932.

OMeara, C., Schlag, L., \& Wickler, M. (2018). Applications of deep learning neural networks to satellite telemetry monitoring. In 2018 SpaceOps Conference (p. 2558). https://doi.org/10.2514/6.2018-2558. 
Parbat, D., \& Chakraborty, M. (2020). A python-based support vector regression model for prediction of COVID19 cases in India. Chaos, Solitons \& Fractals, 138, 109942. https://dx.doi.org/10.1016\%2Fj.chaos.2020.109942.

Pietromonaco, P. R., \& Overall, N. C. (2020). Applying relationship science to evaluate how the COVID-19 pandemic may impact couples' relationships. American Psychologist. http://dx.doi.org/10.1037/amp0000714.

Roy, S., Bhunia, G. S., \& Shit, P. K. (2021). Spatial prediction of COVID-19 epidemic using ARIMA techniques in India. Modeling earth systems and environment, 7(2), 1385-1391. https://doi.org/10.1007/s40808-020-00890-y.

Rustam, F., Reshi, A. A., Mehmood, A., Ullah, S., On, B. W., Aslam, W., \& Choi, G. S. (2020). COVID-19 future forecasting using supervised machine learning models. IEEE access, 8, 101489101499. https://doi.org/10.1109/ACCESS.2020.2997311.

Sotgiu, G., \& Dobler, C. C. (2020). Social stigma in the time of coronavirus disease 2019. Eur Respir J, 56: 2002461https://doi.org/10.1183/13993003.02461-2020.

Stankovska, G., Memedi, I., \& Dimitrovski, D. (2020). Coronavirus COVID-19 disease, mental health and psychosocial support. Society Register, 4(2), 33-48. https://doi.org/10.14746/sr.2020.4.2.03.

Salimi, R., Gomar, R., \& Heshmati, B. (2020). The COVID-19 outbreak in Iran. Journal of global health, 10(1). https://dx.doi.org/10.7189\%2Fjogh.10.010365.

Shalbafan, M., \& Khademoreza, N. (2020). What we can learn from COVID-19 outbreak in Iran about the importance of alcohol use education. The American journal of drug and alcohol abuse, 46(3), 385-386. https://doi.org/10.1080/00952990.2020.1753759.

Sazli, M. H. (2006). A brief review of feed-forward neural networks. Communications Faculty of Sciences University of Ankara Series A2-A3 Physical Sciences and Engineering, 50(01). https://doi.org/10.1501/0003168.

Shaharudin SM, Ismail S, Hassan NA, Tan ML and Sulaiman NAF. (2021). Short-Term Forecasting of Daily Confirmed COVID-19 Cases in Malaysia Using RF-SSA Model. Frontiers in public health Health 9:604093. https://doi.org/10.3389/fpubh.2021.604093.

Satrio, C. B. A., Darmawan, W., Nadia, B. U., \& Hanafiah, N. (2021). Time series analysis and forecasting of coronavirus disease in Indonesia using ARIMA model and PROPHET. Procedia Computer Science, 179, 524-532. https://doi.org/10.1016/ELSEVIER_CM_POLICY.

Tamang, S. K., Singh, P. D., \& Datta, B. (2020). Forecasting of Covid-19 cases based on prediction using artificial neural network curve fitting technique. Global Journal of Environmental Science and Management, 6(Special Issue (Covid-19)), 53-64. DOI: 10.22034/gjesm.2020.04.0.

Turjman, F. (2021). COVID-19 cases prediction by using hybrid machine learning and beetle antennae search approach. Sustainable Cities and Society, 66, 102669. https://dx.doi.org/10.1016\%2Fj.scs.2020.102669. 
Talkhi, N., Fatemi, N. A., Ataei, Z., \& Nooghabi, M. J. (2021). Modeling and forecasting number of confirmed and death caused COVID-19 in IRAN: A comparison of time series forecasting methods. Biomedical Signal Processing and Control, 66, 102494. https://dx.doi.org/10.1016\%2Fj.bspc.2021.102494.

Takian, A., Raoofi, A., \& Kazempour-Ardebili, S. (2020). COVID-19 battle during the toughest sanctions against Iran. Lancet (London, England), 395(10229), 1035. https://dx.doi.org/10.1016\%2FS0140-6736(20)30668-1.

Verma, A., \& Prakash, S. (2020). Impact of covid-19 on environment and society. Journal of Global Biosciences, 9(5), 7352-7363. https://ssrn.com/abstract=3644567.

Worldometers dataset. 2021. Announced new COVID-19 deaths by country. Available at: https://www.worldometers.info/coronavirus/\#countries.

Workie, E., Mackolil, J., Nyika, J., \& Ramadas, S. (2020). Deciphering the impact of COVID-19 pandemic on food security, agriculture, and livelihoods: A review of the evidence from developing countries. Current Research in Environmental Sustainability, 100014. https://doi.org/10.1016/j.crsust.2020.100014.

Wahid, F., Alsaedi, A. K. Z., \& Ghazali, R. (2019). Using improved firefly algorithm based on genetic algorithm crossover operator for solving optimization problems. Journal of Intelligent \& Fuzzy Systems, 36(2), 1547-1562. DOI: 10.3233/JIFS-181936.

Xiong, J., Lipsitz, O., Nasri, F., Lui, L. M., Gill, H., Phan, L., ... \& McIntyre, R. S. (2020). Impact of COVID-19 pandemic on mental health in the general population: A systematic review. Journal of affective disorders. https://doi.org/10.1016/j.jad.2020.08.001.

Yoosefi Lebni, J., Abbas, J., Moradi, F., Salahshoor, M. R., Chaboksavar, F., Irandoost, S. F., ... \& Ziapour, A. (2021). How the COVID-19 pandemic effected economic, social, political, and cultural factors: A lesson from Iran. International Journal of Social Psychiatry, 67(3), 298-300. https://doi.org/10.1177/0020764020939984.

Yeh, W. C., \& Hsieh, T. J. (2012). Artificial bee colony algorithm-neural networks for S-system models of biochemical networks approximation. Neural Computing and Applications, 21(2), 365375. https://doi.org/10.1007/s00521-010-0435-z.

Yu, C. S., Chang, S. S., Chang, T. H., Wu, J. L., Lin, Y. J., Chien, H. F., \& Chen, R. J. (2021). A COVID-19 Pandemic Artificial Intelligence-Based System with Deep Learning Forecasting and Automatic Statistical Data Acquisition: Development and Implementation Study. Journal of medical Internet research, 23(5), e27806. https://doi.org/10.2196/27806.

Yang, X. S., \& He, X. (2013). Firefly algorithm: recent advances and applications. International journal of swarm intelligence, 1(1), 36-50. https://doi.org/10.1504/IJSI.2013.055801.

Zhou, G., Moayedi, H., Bahiraei, M., \& Lyu, Z. (2020). Employing artificial bee colony and particle swarm techniques for optimizing a neural network in prediction of heating and cooling 
loads of residential buildings. Journal of Cleaner Production, 254, 120082. https://doi.org/10.1016/j.jclepro.2020.120082.

Zivkovic, M., Bacanin, N., Venkatachalam, K., Nayyar, A., Djordjevic, A., Strumberger, I., \& AlTurjman, F. (2021). COVID-19 cases prediction by using hybrid machine learning and beetle antennae search approach. Sustainable Cities and Society, 66, 102669. https://dx.doi.org/10.1016\%2Fj.scs.2020.102669.

Zheng, N., Du, S., Wang, J., Zhang, H., Cui, W., Kang, Z., ... \& Xin, J. (2020). Predicting COVID19 in China using hybrid AI model. IEEE transactions on cybernetics, 50(7), 2891-2904. https://doi.org/10.1109/TCYB.2020.2990162.

Zabihi, F., \& Nasiri, B. (2018). A novel history-driven artificial bee colony algorithm for data clustering. Applied Soft Computing, 71, 226-241. https://doi.org/10.1016/j.asoc.2018.06.013.

Zorlu, K., Gokceoglu, C., Ocakoglu, F., Nefeslioglu, H. A., \& Acikalin, S. J. E. G. (2008). Prediction of uniaxial compressive strength of sandstones using petrography-based models. Engineering Geology, 96(3-4), 141-158. http://dx.doi.org/10.1016/j.enggeo.2007.10.009. 